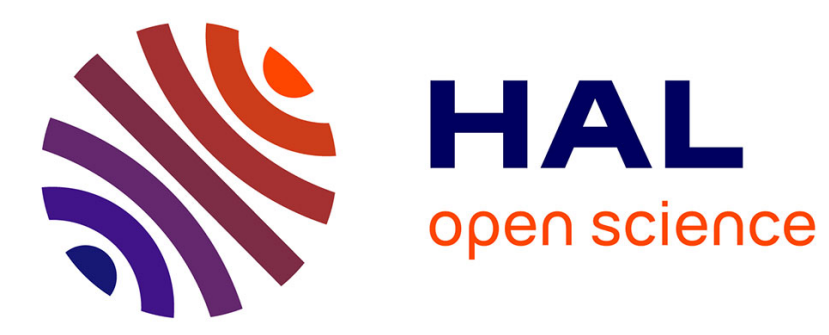

\title{
La centrale expérimentale Thémis : bilan et perspectives
}

B. Bonduelle, B. Rivoire, A. Ferriere

\section{To cite this version:}

B. Bonduelle, B. Rivoire, A. Ferriere. La centrale expérimentale Thémis: bilan et perspectives. Revue de Physique Appliquée, 1989, 24 (4), pp.453-461. 10.1051/rphysap:01989002404045300 . jpa00246067

\section{HAL Id: jpa-00246067 https://hal.science/jpa-00246067}

Submitted on 1 Jan 1989

HAL is a multi-disciplinary open access archive for the deposit and dissemination of scientific research documents, whether they are published or not. The documents may come from teaching and research institutions in France or abroad, or from public or private research centers.
L'archive ouverte pluridisciplinaire HAL, est destinée au dépôt et à la diffusion de documents scientifiques de niveau recherche, publiés ou non, émanant des établissements d'enseignement et de recherche français ou étrangers, des laboratoires publics ou privés. 
Classification

Physics Abstracts

$86.10 \mathrm{~K}-86.30$

\title{
La centrale expérimentale Thémis : bilan et perspectives
}

\author{
B. Bonduelle, B. Rivoire et A. Ferriere \\ Institut de Science et de Génie des Matériaux et Procédés, C.N.R.S., B.P. n 5, Odeillo, 66120 Font Romeu, \\ France
}

(Reçu le 20 mai 1988, révisé le 7 novembre 1988, accepté le 16 décembre 1988)

\begin{abstract}
Résumé. - L'ensemble des résultats d'expérimentation obtenus sur le site de la centrale solaire Thémis est présenté. Si la production globale du prototype n'est pas significative, son fonctionnement expérimental a permis d'obtenir les lois de rendement de chaque sous-système, de proposer des améliorations techniques et de valider une simulation permettant d'évaluer la production de la filière. Une centrale industrielle de plus grande taille peut produire $245 \mathrm{kWh} / \mathrm{m}^{2}$. an en France et $330 \mathrm{kWhe} / \mathrm{m}^{2}$. an en Californie avec un rendement de conversion global annuel de $14 \%$.
\end{abstract}

\begin{abstract}
The experimental results of Thémis, the french central receiver solar power plant, are presented : the measured overall production of the pilot plant was not significant but its testing operation gave the conversion laws of each sub-systems and brought lessons for technical improvements. A simulation based on these results was performed to evaluate the potential production of a larger industrial plant : $245 \mathrm{kWh} / \mathrm{m}^{2}$.year can be produced in france and $330 \mathrm{kWh} / \mathrm{m}^{2}$.year in California, with a conversion ratio of $14 \%$.
\end{abstract}

\section{Introduction.}

Quand, en 1975, le projet d'une centrale solaire à tour française a été mis en chantier, par EDF et le CNRS, le but visé était d'en étudier la faisabilité technique et de poser un jalon sur la voie d'une maîtrise des coûts.

La phase d'exploitation de la centrale pilote Thémis étant maintenant achevée, un bilan de cette expérience peut être établi. Cet article, après avoir rappelé l'historique et le descriptif du projet, présente un résumé de l'ensemble des résultats acquis et les conclusions des extrapolations qui ont pu être faites sur la base de ceux-ci pour évaluer les performances d'une installation de qualité industrielle qu'il serait aujourd'hui possible de concevoir.

On y verra que, contrairement à une opinion parfois exprimée, la mise en œuvre de ce prototype a permis de répondre très largement aux questions posées au départ et de définir avec précision les possibilités futures de la filière.

Le travail présenté ici est un résumé des travaux réalisés par l'équipe G.E.S.T. (groupe d'évaluation scientifique Thémis), équipe mixte CNRS-AFME implantée sur le site de Thémis et rattachée en 1983 au laboratoire d'Odeillo. La synthèse de ces travaux a fait l'objet d'un rapport interne [1] et de publications disponibles au Centre de Documentation Scientifique du C.N.R.S.

\section{La centrale solaire.}

2.1 DESCRIPTION (VOIR PHOTO). - Lancé au lendemain de la crise du pétrole par le CNRS pour étudier la faisabilité du concept de centrale solaire à tour, le programme THEM (Thermo-Hélio-ElectriqueMégawatts) a mené à la construction de la centrale expérimentale Thémis [2-5].

La figure 1 et l'annexe donnent les éléments caractéristiques du prototype [6]. Il faut souligner les options principales qui font sa spécificité :

- le choix du fluide caloporteur, un mélange de sels fondus, était le choix technologique le plus risqué. Les avantages escomptés de souplesse en fonctionnement par rapport au choix classique de l'eau/vapeur pouvaient être anéantis par les problèmes technologiques posés par la solidification du sel à $140^{\circ} \mathrm{C}$;

- récepteur solaire à cavité, échangeur à rayon- 


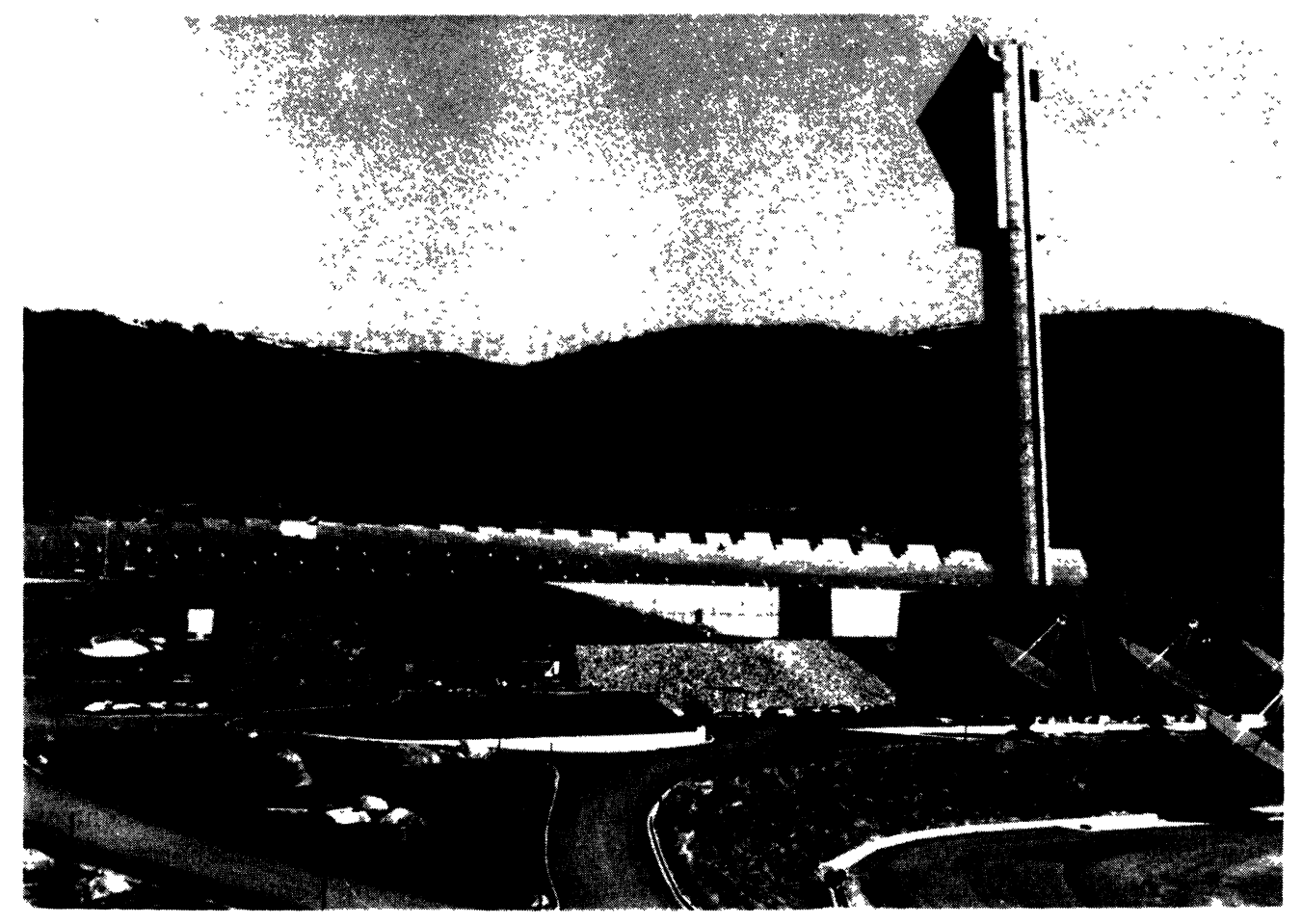

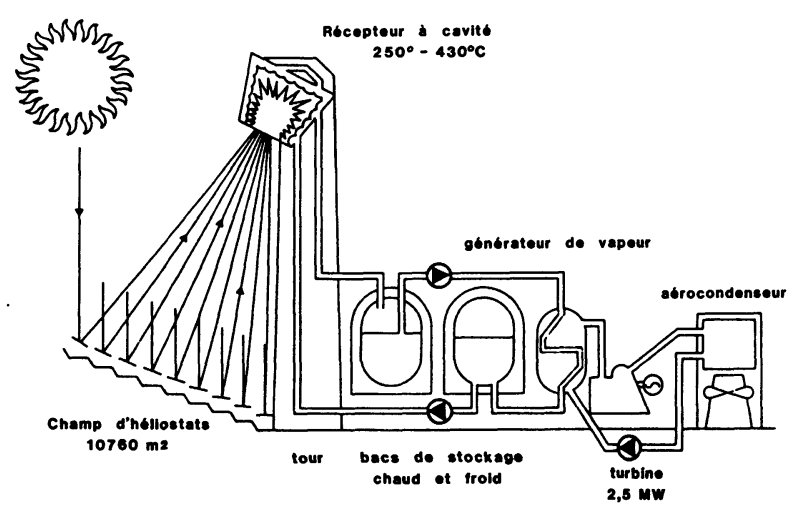

Fig. 1. - Schéma de principe de la centrale Thémis.

[Themis schematic diagram.]

nement de grande taille dont les parois sont tapissées de tubes jointifs ;

- stockage thermique important utilisant la chaleur sensible des sels fondus répartis en 2 bacs assurant l'indépendance entre le récepteur (boucle primaire) et la production d'électricité (boucle secondaire).

D'autres dispositions secondaires constituaient également des prototypes : source froide par aérocondenseur sec à tirage naturel, dispositif auxiliaire de production de chaleur par capteurs solaires paraboliques (type THEK), contrôle commande informatisé.
2.2 LES BUTS DE L'EXPÉRIMENTATION. - Le programme d'expérimentation s'articulait autour de trois axes :

- démonstration de la faisabilité de la filière à sel fondu et étude détaillée des composants spécifiques (champ d'héliostat, récepteur et stockage);

- construction d'outils d'ingénierie performants et validés pour la conception et l'étude des centrales solaires : il s'agit surtout de trois modèles permettant d'effectuer le calcul du comportement thermique du récepteur et l'optimisation de l'ensemble champchaudière ;

- analyse du bilan global et des données d'exploitation pour qualifier la filière et connaître sa productibilité. Cette analyse nécessite de préciser de nouveaux choix technologiques et d'optimiser/automatiser la conduite de la centrale afin d'apporter des améliorations technico-économiques significatives.

\section{Les performances globales mesurées.}

3.1 FAISABILITÉ ET FONCTIONNEMENT. - La figure 2 montre l'enregistrement des puissances solaire incidente sur le champ et absorbée par le récepteur, brute et nette électrique ainsi que l'évolution de l'énergie stockée dans les 2 bacs.

Ce fonctionnement observé lors d'une belle journée démontre la faisabilité du concept pendant une journée. On constate la très grande souplesse de marche du récepteur, la rapidité du démarrage matinal et un suivi correct des passages nuageux [7]. 


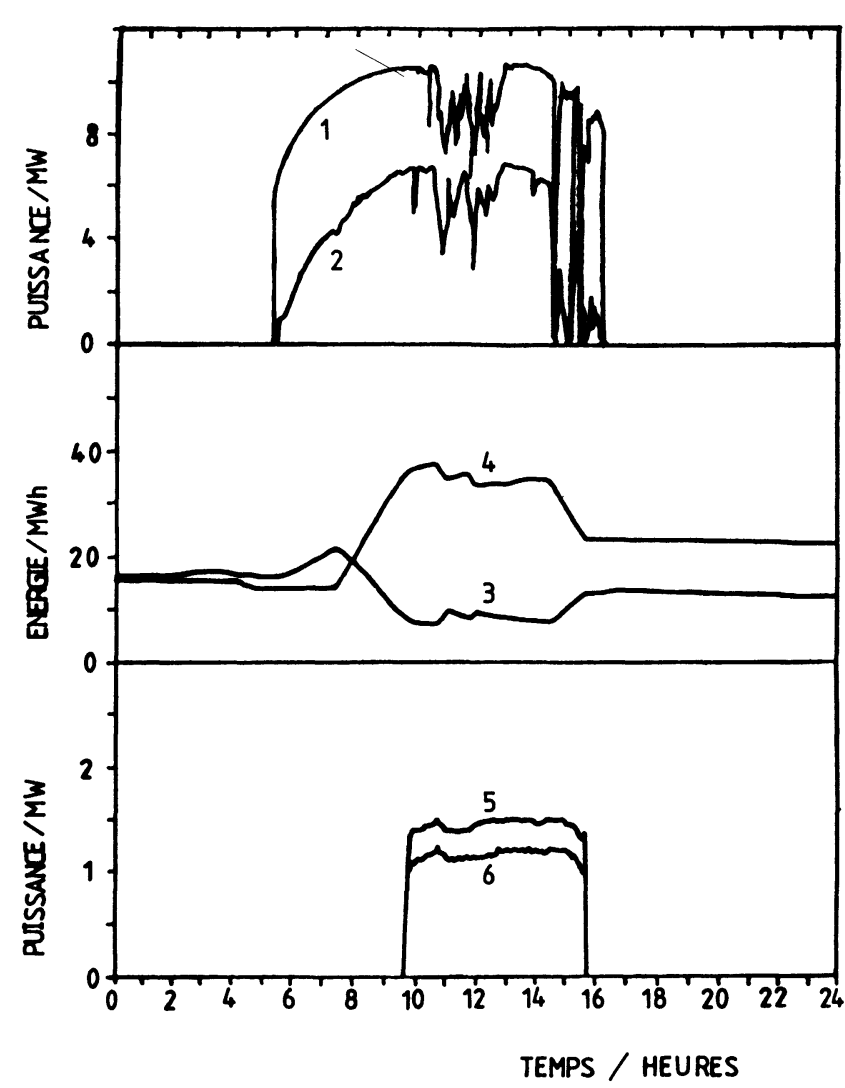

Fig. 2. - Enregistrement des puissances solaire incidente sur le champ (1), absorbée par le récepteur (2), électrique brute (5) et nette produite (6), et évolution de l'énergie stockée dans le bac froid (3) et chaud (4) pendant la journée du 6 juin 1986.

[Direct insolation (1), power absorbed in the receiver (2), energy stored in the cold (3) or the hot (4) tank, gross electric (5) and net electric (6) power output measured on june 6th, 1986.]

\subsection{Puissances AUX CONDITIONS NOMINALES-} COMPARAISON AVEC LE PROJET. - L'analyse de la chaîne de conversion à midi lors d'une belle journée (18 avril 1985) nous fournit la décomposition décrite dans le tableau I. On obtient un rendement brut (production de l'alternateur/puissance solaire incidente) de 0,19 aux conditions nominales et un rendement net (puissance fournie au réseau/puissance solaire incidente) de 0,17 .

Le faible écart de ces résultats par rapport au projet provient surtout du groupe turbo-alternateur qui ne fonctionne pas à la température prévue, le sel n'étant pas chauffé à $450^{\circ} \mathrm{C}$.

Donc à la puissance nominale, Thémis est très proche des caractéristiques du projet : cela est une vérification a posteriori des principes ayant présidé aux dimensionnements de l'installation et de la qualité de la réalisation.

3.3 BILAN JOURNALIER. - Mais ces rendements ne peuvent être atteints sur toute une journée compte
Tableau I. - Thémis. Comparaison des performances en puissance mesurées le 18 avril 1985 à midi avec celles du projet au point nominal.

[Comparison between designed and measured performances at nominal conditions.]

\begin{tabular}{|c|c|c|c|}
\hline \multirow{2}{*}{ Sous-systèmes } & \multicolumn{3}{|c|}{$\begin{array}{l}\text { Rendement en puissance } \\
\text { au point nominal }\end{array}$} \\
\hline & Projet & \multicolumn{2}{|c|}{ Mesuré } \\
\hline $\begin{array}{c}\text { Ensoleillement } \\
\text { Météo : W/m² } \\
\text { Champ : } \mathrm{kW}\end{array}$ & $\begin{array}{r}1040 \\
11200\end{array}$ & \multicolumn{2}{|c|}{1040} \\
\hline $\begin{array}{l}\text { Champ d'héliostats } \\
\text { Réflexion } \\
\text { Surface efficace } \\
\text { Disponibilité } \\
\text { Taux d'utilisation }\end{array}$ & $\begin{array}{l}0,90 \\
0,94 \\
1 \\
-\end{array}$ & $\begin{array}{r}\text { Rend } \\
\\
0,90 \\
0,96 \\
0,99\end{array}$ & $\begin{array}{c}\text { Pertes } \\
(\mathrm{kW})\end{array}$ \\
\hline $\begin{array}{l}\text { Récepteur } \\
\text { Interception } \\
\text { Réflexion visible } \\
\text { Emission IR } \\
\text { Convection } \\
\text { Conduction } \\
\text { Gestion porte }\end{array}$ & $\begin{array}{l}0,98 \\
0,96\end{array}$ & $\begin{array}{l}0,98 \\
0,99\end{array}$ & $\begin{array}{r}230 \\
140 \\
30\end{array}$ \\
\hline $\begin{array}{l}\text { Transfert-stockage } \\
\text { Boucle primaire } \\
\text { Stockage } \\
\text { Boucle secondaire } \\
\text { Conditionnement }\end{array}$ & 0,98 & & $\begin{array}{l}57 \\
63 \\
13\end{array}$ \\
\hline $\begin{array}{l}\text { Turbo-alternateur } \\
\text { Cycle }\end{array}$ & 0,28 & 0,25 & \\
\hline $\begin{array}{l}\text { Auxiliaires } \\
\text { - Principaux } \\
\text { Champ } \\
\text { Pompes sel } \\
\text { Traçage sel } \\
\text { Secondaire } \\
\text { - Généraux }\end{array}$ & 0,90 & & $\begin{array}{r}10 \\
70 \\
3 \\
71 \\
60\end{array}$ \\
\hline $\begin{array}{c}\text { Rendement global } \\
\text { Brut } \\
\text { Net }\end{array}$ & $\begin{array}{l}0,22 \\
0,20\end{array}$ & $\begin{array}{l}0,19 \\
0,17\end{array}$ & \\
\hline
\end{tabular}

tenu de la variation de la puissance réfléchie par le champ ainsi que des consommations des auxiliaires et de l'énergie nécessaire pour les maintiens à chaud lors des arrêts.

La figure 3 donne le diagramme des énergies dans la chaîne de conversion pour la journée du 18 avril 1985. Le rendement brut atteint 0,14 mais le rendement énergétique net tombe à 0,095 . 


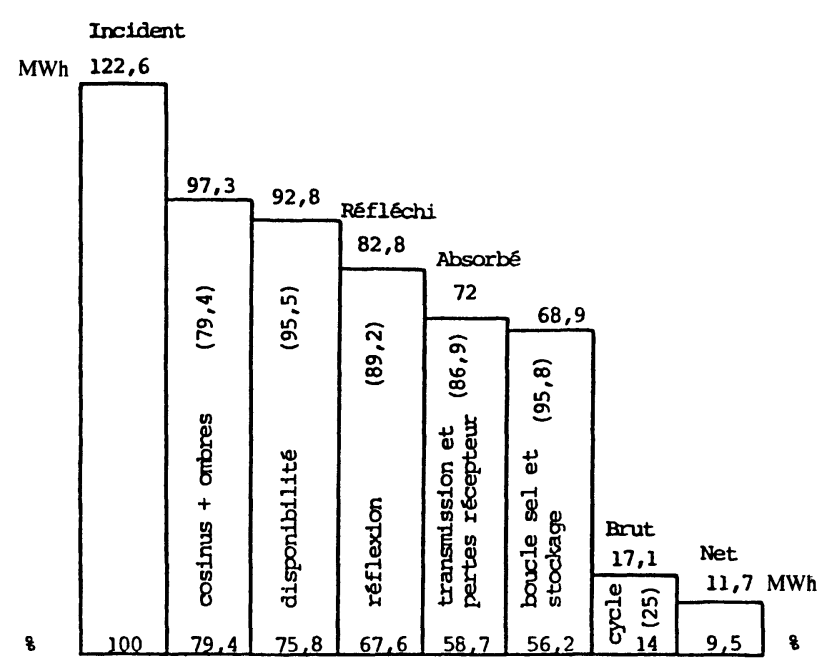

Fig. 3. - Diagramme des énergies et rendements dans la chaîne de conversion hélio-thermo-électrique.

[Daily energy production and subsystems efficiencies.]

3.4 BILAN ANNUEL : NÉCESSITÉ D’UNE ESTIMATION DE LA PRODUCTION. - Sur l'ensemble de l'année, le rendement de Thémis chute encore plus : par rapport à une belle journée, les fonctionnements à charge partielle (ensoleillement plus faible) réduisent les rendements moyens de conversion et les arrêts augmentent la part relative de consommation des auxiliaires.

Ainsi, par exemple, la puissance moyenne absorbée au récepteur, mesurée sur une longue période, est de 4,5 MW au lieu de $6 \mathrm{MW}$ en moyenne pour la journée de la figure 3 et de $9 \mathrm{MW}$ aux conditions nominales.

On ne peut donc pas extrapoler le bilan journalier précédent pour évaluer la production annuelle de la centrale.

Par ailleurs, la production mesurée à Thémis sur une longue durée n'est pas significative. En tant que prototype expérimental, Thémis a subi de nombreux arrêts consécutifs à des mises au point ou avaries : sur une période de 3 ans, Thémis a été arrêtée pendant une durée supérieure à une année, et n'a jamais fonctionné pendant les mois d'été. Les procédures d'exploitation, elles-mêmes expérimentales, ont rarement permis un fonctionnement pour lequel les consommations auxiliaires soient minimisées. On ne peut donc pas se baser sur la production globale de Thémis pour évaluer les possibilités de la filière.

Il a donc été nécessaire de développer une méthode pour estimer la production potentielle de Thémis et de sa filière. Comme la centrale n'a jamais fonctionné correctement, il est difficile de faire une estimation qui soit à la fois réaliste et représentative d'une installation fiable.

La méthode de la caractéristique proposée par Izygon [8], qui a travaillé sur nos données, a l'avantage d'être facilement généralisable à d'autres centrales ou à d'autres données météorologiques. Mais comme elle s'appuie sur une analyse statistique des mesures d'exploitation, elle reste proche de résultats bruts du prototype et ne permet pas d'estimer la production d'une installation corrigée de ses défauts de conception.

Nous avons choisi une méthode $[9,10]$ où l'on part de l'énergie solaire reçue annuellement par le champ et de laquelle on retranche les pertes des divers soussystèmes successifs dans la chaîne de transformation thermodynamique.

Pour une estimation réaliste nous utilisons les lois de comportement observées pendant les périodes de bon état de marche de chaque sous-système, et les pertes annuelles sont obtenues à partir d'un traitement détaillé des données météorologiques d'une année.

Pour justifier l'hypothèse d'un bon fonctionnement de l'ensemble, il a fallu procéder à une analyse technologique des incidents et proposer des solutions réalistes aux défauts rencontrés.

C'est la synthèse des résultats de la méthode que nous présentons ici.

\section{Lois de pertes des sous-systèmes.}

L'étude détaillée de chaque composant a demandé la mise en œuvre d'un ensemble de techniques et de méthodes expérimentales très diversifiées.

Nous donnons ci-dessous quelques éléments pour illustrer les méthodes utilisées et mettre en valeur certains résultats mais nous ne pouvons pas développer l'ensemble des mesures dans le cadre de cette note de synthèse.

4.1 LE CHAMP D'HÉLIOSTATS. - Le rendement du champ dépend du coefficient de réflexion global, de la surface efficace (effet cosinus et ombres), du nombre d'héliostats disponibles et des qualités de pointage et de réglage de ceux-ci $[11,12]$ :

- le coefficient de réflexion global est obtenu à partir d'un échantillonnage de 108 points de mesure de la réflectivité des miroirs. Effectuée chaque semaine, cette série de mesure nous a conduit à un facteur moyen de réflexion annuel de 0,83 ;

- la surface efficace est déterminée par un calcul géométrique recalé sur des mesures d'ombres portées : sur l'année, on constate que $18 \%$ de l'énergie solaire incidente sur les miroirs est perdue par effet cosinus ou par les ombres ;

- pour qualifier le pointage et le réglage des héliostats, nous avons analysé plus de 600 distributions des flux réfléchis par les héliostats acquises par une cible de 1024 photocellules. Ces mesures nous ont aussi permis de mettre au point un code de calcul des flux réfléchis par le champ vers le récepteur, utile pour faire le bilan thermique de ce dernier. 
Ces résultats amènent à un rendement moyen annuel du champ, rapport entre l'énergie renvoyée par le champ et l'énergie solaire incidente sur celuici, de 0,61 .

Des améliorations sur la disponibilité et la réflectivité des miroirs permettraient de l'augmenter à 0,70 .

4.2 LE RÉCEPTEUR SOlAIRE. - Il est difficile de dissocier expérimentalement les différentes pertes, aussi nous faisons appel à différentes méthodes complémentaires pour les calculer [12, 14].

Les pertes par réflexion s'élèvent à près de $1 \%$ de la puissance solaire entrant dans le récepteur. Ce chiffre résulte de :

- la connaissance du facteur d'absorption du revêtement des tubes constituant la paroi interne;

- de l'effet de macrorugosité de ces tubes;

- et du bilan radiatif global dans la cavité obtenu à l'aide d'un modèle où le récepteur est découpé en facettes dont on calcule les équilibres radiatifs à partir de la répartition des flux solaires entrants, calculés par le code signalé au paragraphe précédent.

Avec un modèle analogue, les pertes par émission sont obtenues à partir de la distribution des températures et de l'émissivité de la paroi. Cette dernière a été mesurée à l'aide d'une caméra infra-rouge. Au régime nominal de fonctionnement du récepteur (cf. annexe), les pertes émissives s'élèvent à $230 \mathrm{~kW} \mp$ $20 \mathrm{~kW}$.

Les pertes conductives du récepteur, qui sont faibles en régime permanent sous flux, peuvent devenir importantes en régime transitoire. Un modèle de conduction à travers les parois et l'isolant, $\mathrm{du}$ récepteur et de sa porte, a été recalé sur des essais de refroidissement du récepteur porte fermée

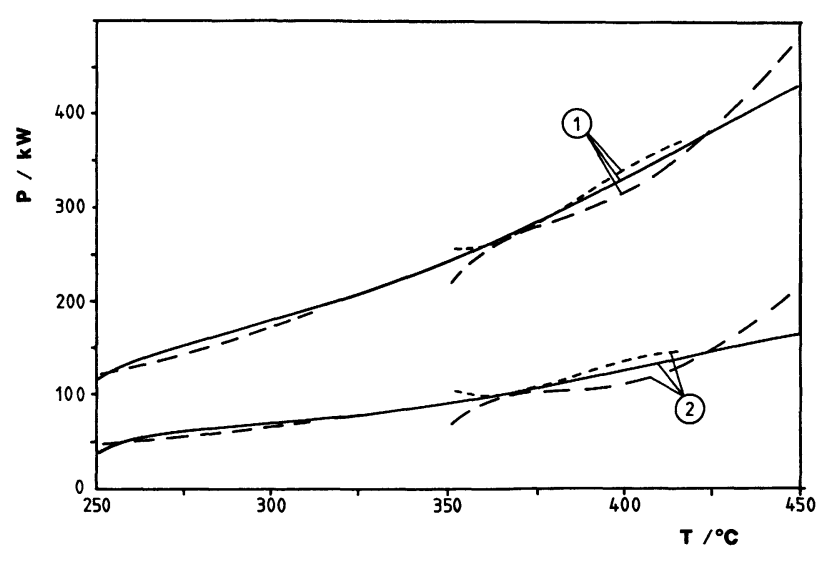

Fig. 4. - Pertes totales (1) et convectives (2) du récepteur en fonction de sa température, $\mathrm{T}$. Chaque courbe correspond à un essai en recirculation: 20/1/86 (- $\longrightarrow$ ), 17/12/85 (- — - 2/12/85 (- - - -).

[Total thermal (1) and convective (2) losses of the receiver versus its temperature, each line corresponds to a recirculating test.] par identification des résistances thermiques des parois.

Pour les pertes convectives, nous avons procédé à des essais de refroidissement du récepteur, porte ouverte sans flux solaire, le fluide était maintenu en circulation afin que le récepteur soit quasiment isotherme.

Les pertes totales à une température donnée sont alors proportionnelles à la pente de la courbe de refroidissement à cette température. On déduit les pertes convectives en retranchant les pertes conductives et émissives évaluées comme indiqué ci-dessus.

La figure 4 donne les pertes totales mesurées ainsi que les pertes convectives (sans vent) en fonction de la température du récepteur supposé isotherme.

Enfin, le traitement des données acquises en fonctionnement aux conditions nominales de température du récepteur, donne le rendement global du récepteur en fonction de la puissance réfléchie par le champ $P_{\text {ref }}$ (Fig. 5). Ce rendement peut être approché par la loi simplifiée suivante :

$$
\eta=\frac{0,96 P_{\text {ref }}-400}{P_{\text {ref }}},
$$

où $P_{\text {ref }}$ est exprimée en $\mathrm{kW}$.

Le terme constant, $400 \mathrm{~kW}$, représente les pertes convectives, conductives et émissives. Le terme proportionnel, 0,96 représente le produit du rendement de réflexion du récepteur, du rendement d'ouverture et du rendement de transfert champrécepteur (absorption atmosphérique).

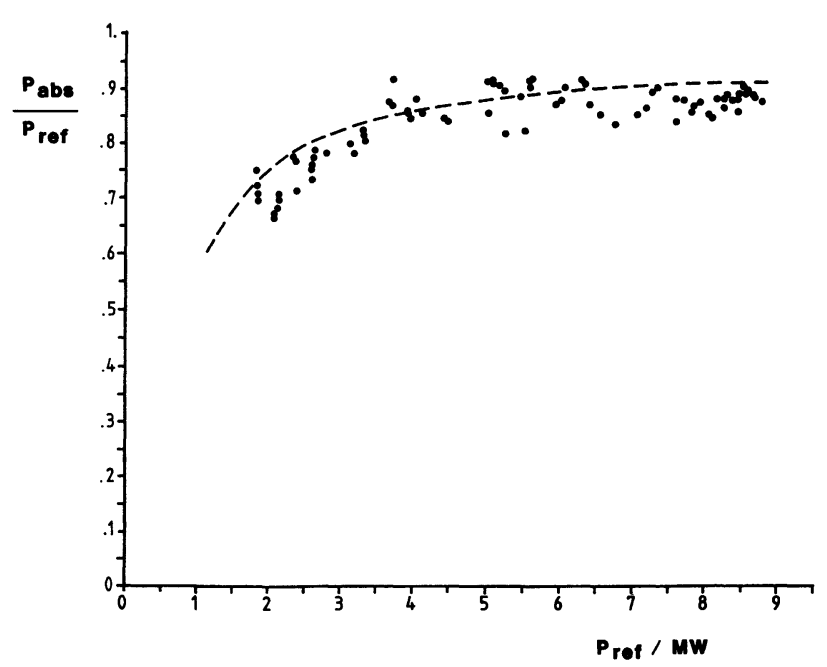

Fig. 5. - Rendement du récepteur (puissance absorbée/puissance réfléchie par le champ) en fonction de la puissance réfléchie par le champ, aux conditions nominales de fonctionnement définies en annexe. Chaque point correspond à une mesure sur une période stable de $10 \mathrm{~min}$.

[Receiver efficiency versus reflected solar power at nominal conditions. One point represents a $10 \mathrm{~min}$ stable period.] 
4.3 Autres sous-SYSTÈmES. - L'étude des bacs de stockage [15] nous a donné une loi des pertes thermiques totales des bacs en fonction de leur température. Au régime nominal (bac froid à $250^{\circ}$ et bac chaud à $430^{\circ} \mathrm{C}$ ) la puissance perdue par les bacs s'élève à $63 \mathrm{~kW}$.

Enfin, la modélisation complète des boucles thermiques, et la validation de ce modèle, nous ont permis de calculer avec une bonne précision le coût énergétique des refroidissements-réchauffages nocturnes ou nébulaires et d'identifier la conduite optimale de ces boucles à l'arrêt $[16,17]$.

\section{Enseignement technologique. Améliorations pro- posées.}

L'essentiel des incidents a été surtout occasionné par le matériel conventionnel (mise au point des automates, avarie du transformateur et de l'alternateur par défaut des protections, ...). Leur analyse [18] a toujours permis de trouver des solutions réalistes qui ne seront pas décrites ici. Les composants spécifiquement solaires (héliostats, récepteur) se sont bien comportés.

Pour le champ d'héliostats, la modification de deux composants (codeurs inductifs, batteries) permettrait de réduire des $2 / 3$ la charge d'entretien et accroîtrait la disponibilité.

Le récepteur et le circuit primaire ont eu un excellent comportement : ils n'ont eu à subir ni ruptures, ni corrosion, ce qui était pourtant à craindre. De grosses difficultés ont été rencontrées au niveau du système de préchauffage des tuyauteries, nécessaire pour éviter la solidification du sel et la formation des bouchons.

Les modifications définies [18], ainsi que l'expérience acquise, ont fait faire un grand pas à la technologie d'utilisation des sels fondus : on peut noter à ce propos que les nouveaux projets de centrales solaires mettent en avant les sels fondus par rapport aux autres solutions (eau/vapeur, sodium).

Enfin, pour la conception des boucles thermiques, il faut prendre en compte le caractère cyclique de l'énergie incidente qui multiplie les transitoires et impose des arrêts quotidiens. Pour cela, il y a lieu de minimiser la consommation des auxiliaires à l'arrêt et de simplifier les circuits pour réduire leurs pertes thermiques. Les matériels classiques (robinetterie, joints...) doivent être de qualité supérieure pour résister aux contraintes thermiques répétées. Les cyclages peuvent être réduits par une politique de maintien à chaud systématique que nous préconisons : une étude [16] a en effet montré que dans presque tous les cas le maintien à chaud des tuyauteries pendant les arrêts ne consomme pas plus d'énergie que les séquences de refroidissement-réchauf- fage avec vidange de sel dans le stockage : de plus ce maintien à chaud apporte souplesse et disponibilité.

\section{Ce que Thémis pourrait produire [19].}

6.1 Une CENTRAle Thémis fiable, Thémis A. Dans une première étape, nous avons estimé la production annuelle d'une centrale solaire de conception identique à Thémis, mais ayant une bonne fiabilité, suite à des modifications technologiques mineures ; appelons-la Thémis A.

Le programme de simulation développé en [10] utilise les lois de pertes déduites des mesures faites sur Thémis, et les données météorologiques de l'année 1984, année moyenne.

On suppose en outre que la centrale ne s'arrête qu'une semaine dans l'année, les nombreuses indisponibilités météo permettant d'effectuer les opérations d'entretien courant. Pendant les arrêts quotidiens, les boucles sont maintenues chaudes par la méthode la moins coûteuse qui consiste à les laisser au repos et à renouveler leur niveau de température par de courtes séquences de pompage.

Dans ces conditions, la simulation nous donne une production électrique nette annuelle de $787 \mathrm{MWh}$, soit $73 \mathrm{kWh} / \mathrm{m}^{2}$ de miroir. Cette production qui représente $4 \%$ de l'énergie solaire incidente, aurait pu être atteinte à Thémis si la mise au point avait été menée à son terme. Mais elle est encore décevante.

\subsection{VERS UNE CENTRALE INDUSTRIELlE: THÉMIS} B. - L'analyse des pertes de Thémis A (Fig. 6) et l'expérience acquise nous suggère d'apporter des modifications de conception pour simplifier l'installation et accroître la production.

La première amélioration est de dimensionner les auxiliaires dans le but de réduire leur consommation à l'arrêt. En effet, Thémis, comme les autres centrales solaires, a été dimensionnée pour des conditions nominales fixées (ensoleillement du 21 mars à midi), pour lesquelles les consommations auxiliaires sont secondaires, ce qui n'est plus le cas à l'arrêt. Par exemple, le virage de la turbine à l'arrêt consomme $27 \mathrm{~kW}$ : cela semble négligeable mais cette consommation pendant $7500 \mathrm{~h}$ d'arrêt dans l'année atteint $210 \mathrm{MWh}$, soit le tiers de la production nette de Thémis A! Avec une puissance de $4 \mathrm{~kW}$, qui est suffisante, on aurait économisé $175 \mathrm{MWh}$. De même, la circulation du sel pendant la nuit devrait être assurée par de petites pompes spécifiques.

L'autre poste important de perte est la perte par réflexion des miroirs : on installera des miroirs plus minces, dont la réflectivité atteint $94 \%$.

Enfin, nous simplifierons le tracé de l'installation pour réduire les pertes thermiques qui se produisent en permanence: pour cela nous éliminerons la 


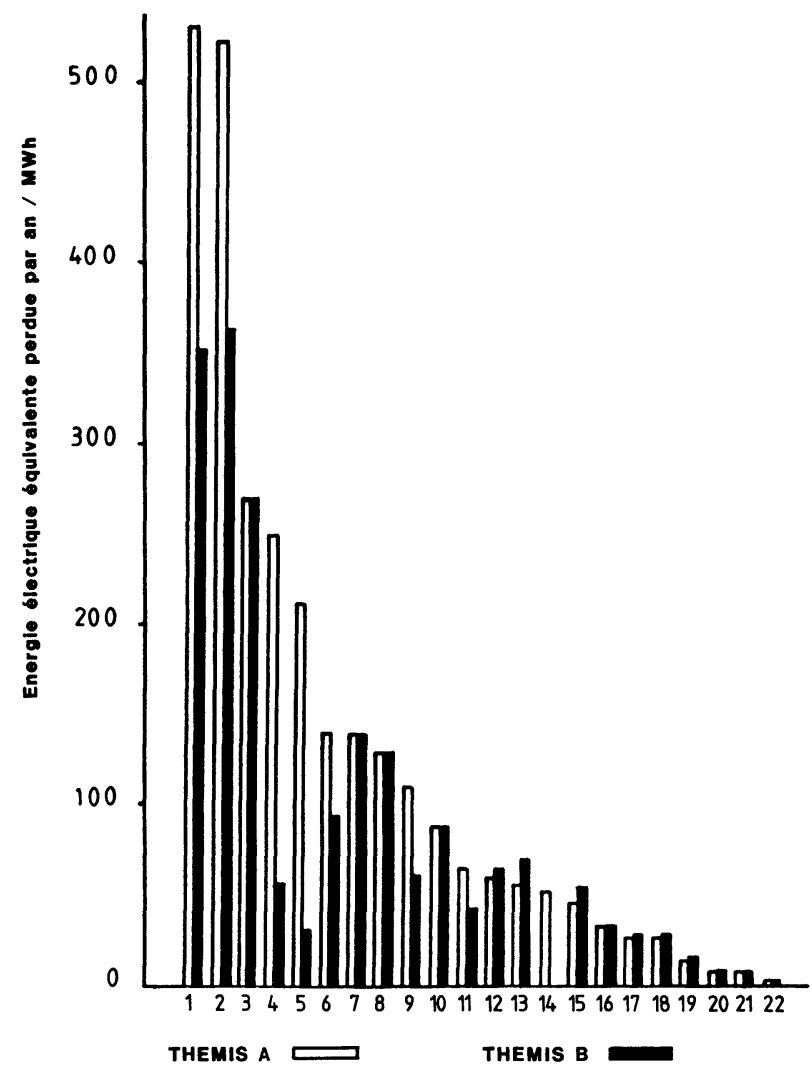

Fig. 6. - Comparaison entre les pertes de Thémis A et Thémis B. Chaque perte est caractérisée par le déficit de production électrique nette qu'elle entraîne : 1) auxiliaires généraux, 2) réflexion champ, 3) pertes thermiques récepteur, 4) indisponibilité champ, 5) virage groupe, 6) conditionnement boucle primaire, 7) pompage boucle primaire, 8) pertes stockage, 9) conditionnement boucle secondaire hors turbine, 10) alimentation champ, 11) débordement flux récepteur, 12) inutilisation champ (météo), 13) auxiliaires groupe, 14) traçage bras morts, 15) temps d'ouverture porte, 16) pertes thermiques circuit boucle primaire, 17) absorption champ-récepteur, 18) réflexion récepteur, 19) pompage sel récepteur, 20) arrêt entretien, 21) conditionnement turbine, 22) pertes thermiques circuit boucle secondaire.

[Comparison between Themis A and Themis B losses. Each loss is reduced to the annual net electric output deficit which it occurs.]

recirculation du récepteur et nous installerons les purges et les évents dans l'isolation du récepteur. On peut aussi supprimer la duplication de nombreux auxiliaires qui assurent une fonction de secours, car l'exploitation discontinue permet l'entretien et le remplacement pendant les arrêts. L'ensemble de ces modifications définit une nouvelle centrale, Thémis B, qui a les mêmes caractéristiques que Thémis, mais de conception améliorée. Sa production atteint $1700 \mathrm{MWh} / \mathrm{an}$, ou $158 \mathrm{kWh} / \mathrm{m}^{2}$ an, au lieu de $787 \mathrm{MWh} /$ an pour Thémis A. Le gain est d'autant plus notable que les modifications sont souvent des simplifications qui entraînent une baisse des coûts d'installation.

6.3 Production attendue des Centrales A SELS FONDUS. - Pour connaître les potentialités de la filière, il nous reste à étudier l'influence de la taille et de l'ensoleillement, donc du site. La simulation permet de faire varier la taille de la centrale en gardant les mêmes proportions que Thémis. L'accroissement de la taille a un effet positif sur le rendement du groupe et sur les pertes du stockage mais a un effet négatif sur l'absorption atmosphérique champ-chaudière, sur la puissance de pompage dans la tour et sur l'énergie nécessaire au préchauffage : l'effet global est positif comme le montre la courbe inférieure de la figure 7. La production annuelle d'une centrale de $25 \mathrm{MWh}$ (10 fois Thémis) situé au même endroit que Thémis passe à $26425 \mathrm{MWh}$, soit $245 \mathrm{kWh} / \mathrm{m}^{2}$ an.

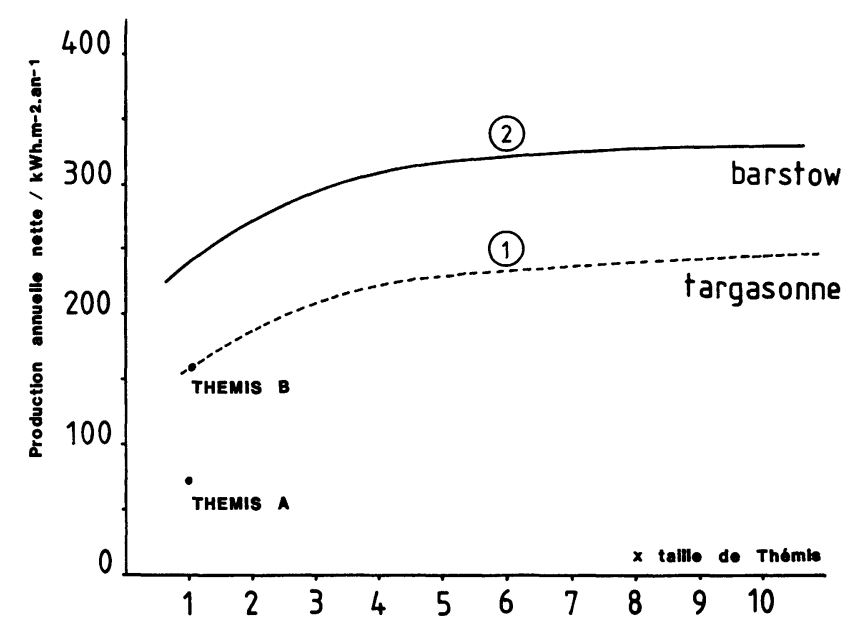

Fig. 7. - Production des centrales de type Thémis en fonction de leur taille situées en France (1), et en Californie (2).

[Themis type solar plants production versus size and climate : south of France (1), California (2).]

La simulation permet aussi de transporter Thémis sous des cieux plus ensoleillés : nous avons utilisé les données météo de la centrale solaire américaine Solar-1 située à Barstow en Californie, où le soleil apporte $2380 \mathrm{kWh} / \mathrm{m}^{2}$.an au lieu de 1700 à Thémis. La production d'une centrale dix fois plus grande que Thémis s'élève alors à $35508 \mathrm{MWh} / \mathrm{an}$, ou $330 \mathrm{kWh} / \mathrm{m}^{2}$.an, avec un rendement net global de $14 \%$, qui devient attractif.

A ce niveau de production le prix de revient du $\mathrm{kWh}$ descend aux alentours de $2 \mathrm{FF}$, ce qui est compétitif dans certains sites ensoleillés et isolés. 
Cela explique que d'autres pays continuent de travailler sur des projets de centrale de taille plus importante, pour lesquels le choix du sel fondu apparaît être le meilleur compromis (projet Phoebus en Europe et ceux des compagnies électriques de l'ouest américain). Certaines de ces études évaluent que le coût du $\mathrm{kWh}$ descendrait à $0,08-0,1 \$(0,5$ à $0,6 \mathrm{FF}$ ) pour une taille de 100 à $200 \mathrm{MWh}$.

\section{Conclusions.}

Nous avons vu que les spécifications du projet et les rendements au régime nominal d'ensoleillement ont été atteints. Le fonctionnement de la centrale se révèle particulièrement souple grâce à l'emploi du sel fondu dans le circuit primaire.

S'agissant d'un prototype, les résultats bruts d'exploitation n'ont pas de signification. Mais l'expérience a permis de rassembler toutes les lois de comportement des sous-systèmes nécessaires à une simulation globale. Celle-ci confirme que Thémis est trop petite pour atteindre une production notable : la taille choisie pour le pilote devait être suffisante pour démontrer la faisabilité et étudier les lois physiques nécessaires à la conception, mais ne pouvait être représentative d'une centrale industrielle. Par contre, grâce aux enseignements acquis, on sait maintenant évaluer avec précision et réalisme ce que donnerait une centrale de taille suffisante dans un site ensoleillé : avec une production annuelle de $330 \mathrm{kWh}$ électrique nette par $\mathrm{m}^{2}$ de miroir et un rendement global annuel de $14 \%$, le concept de centrale à tour est encore bien placé techniquement face aux autres concepts. Le problème réside dans l'évolution des coûts d'installation et d'exploitation dont une évaluation reste à faire.

A l'heure où les recherches sur l'énergie solaire sont abandonnées en France faute d'un marché intérieur, il faut souligner que le bilan de Thémis a été positif : l'expérience a répondu à l'ensemble des questions posées au départ et quelques retombées des techniques de pointe testées sur le site ont eu lieu dans l'industrie.

\section{ANNEXE}

Caractéristiques techniques de Thémis.

\section{HÉLIOSTATS :}

200 héliostats focalisants de $54 \mathrm{~m}^{2}$ chacun, situés au nord de la tour;

- miroir sandwich courbe $2+5 \mathrm{~mm}$, réflectivité $90 \%$;

- champ piloté par calculateur, orientation toutes les $6 \mathrm{~s}$;

- surface au sol du champ : 7 ha ;

- puissance maximale réfléchie par le champ: $9750 \mathrm{~kW}$.

\section{RÉCEPTEUR SOLAIRE :}

- hauteur de la tour : $100 \mathrm{~m}$;

- récepteur de type cavité placé à $80 \mathrm{~m}$;

- volume de la cavité : $56 \mathrm{~m}^{3}$, ouverture carrée (fermée à l'arrêt) ;

- inclinaison du récepteur : $30^{\circ}$ sur l'axe horizontal ;

- fluide caloporteur: sel fondu (mélange de nitrate et de nitrites de potassium et de sodium, appelé « Hitec ») ;

- température d'entrée du Hitec : $250^{\circ} \mathrm{C}$;

- température de sortie du Hitec : $430{ }^{\circ} \mathrm{C}$;

- puissance thermique maximale : $9000 \mathrm{~kW}$.

\section{STOCKAGE :}

- capacité de stockage thermique : $40000 \mathrm{kWh}$ (5 h d'ensoleillement) ;

- fluide de stockage : identique au fluide caloporteur ;

- 2 réservoirs de $300 \mathrm{~m}^{3}$ à $250^{\circ} \mathrm{C}$ et $430{ }^{\circ} \mathrm{C}$.

\section{BOUCLE DE CONVERSION THERMODYNAMIQUE :}

- cycle thermodynamique type Hirn ;

- vapeur surchauffée à $430^{\circ} \mathrm{C}$, pression 50 bars ;

- turbine à 10 étages, $14000 \mathrm{tr} / \mathrm{min}, 4$ soutirages ;

- réfrigération sur aérocondenseur à circulation naturelle ;

- puissance maximale : $2500 \mathrm{~kW}$;

- rendement de conversion : $28 \%$.

\section{Bibliographie}

[1] Bonduelle B., Rivoire B., rapport interne CNRSGEST 01-87 (1987).

[2] Dessus B., Mersier C., Pharabod F., Abatut J. L., Actes du Colloq. l'Electricité Solaire, Toulouse (ed. CNES) 1976, pp. 559.

[3] Chabbal R., Deflandre J., Etievant C., Peube J. L., Rodot M., Courrier du CNRS 23 (1977) 7.
[4] Groupe de projet, rapport interne « Avant-projet THEM $1 »$ CNRS-EDF-THEM-78-08 (1977).

[5] Cosar P., Etievant C., Pouget-Abadie X., Rev. gén. Therm. 200-201 (1978) 627.

[6] Hillairet J., Collon A., Leclerc A., Gravrand J. M., Pouget-AbAdIE X., Entropie 103 (1982) 4. 
[7] Pharabod F., Bezian J. J., Bonduelle B., Rivoire B., Guillard J., Proc. of the 3rd Inter. Workshop on Solar Thermal Central Receiver Systems, Konstanz (ed. Springer Verlag, Berlin), Vol. 1 (1986) 91.

[8] IZYGON M., Thèse, Ecole Centrale Paris (1987).

[9] Boutin V., Rivoire B., Proc. 3rd Inter. Workshop on Solar Thermal Central Receiver Systems, Konstanz (ed. Springer Verlag) Vol. 1 (1986) 383.

[10] Boutin V., Thèse (Nice) 1986.

[11] Bonduelle B., Proc. I.S.E.S. Congress, Montréal (ed. Pergamon Press) 1985.

[12] Bezian J. J., Bonduelle B., Proc. of the 2nd Inter. Workshop on Thermo-mechanical Solar Power Plants, Varese (ed. Reidel, Dordrecht) Vol. 2 (1984) 106.

[13] Bonduelle B., Cazin A. M., Proc. of the 3rd Inter. Workshop on Solar Thermal Central Receiver Systems, Konstanz (ed. Springer Verlag) Vol. 1 (1986) 273.
[14] CaZIN A. M., Thèse, Paris VI (1986).

[15] RIVOIRE B., rapport interne CNRS-GEST-039 (1987).

[16] Ferriere A., Boutin V., Proc. of the 3rd Inter. Workshop on Solar Thermal Central Receiver Systems, Konstanz (ed. Springer-Verlag) Vol. 1 (1986) 317.

[17] Ferriere A., Thèse (Perpignan) 1987.

[18] Bonduelle B., Rivoire B., Proc. of the 3rd Inter. Workshop on Solar Thermal Central Receiver Systems., Konstanz (ed. Springer-Verlag) Vol. 1 (1986) 307.

[19] Bezian J. J., Bonduelle B., Rivoire B., Proc. of I.S.E.S. Congress, Hamburg (ed. Pergamon Press) 1987. 